\title{
MUSE observations of comet 67P/Churyumov-Gerasimenko
}

\section{A reference for future comet observations with MUSE}

\author{
C. Opitom ${ }^{1,2}$, A. Guilbert-Lepoutre ${ }^{3}$, S. Besse ${ }^{4}$, B. Yang ${ }^{1}$, and C. Snodgrass ${ }^{2}$ \\ ${ }^{1}$ ESO (European Southern Observatory) - Alonso de Cordova 3107, Vitacura, Santiago, Chile \\ 2 Institute for Astronomy, University of Edinburgh, Royal Observatory, Edinburgh EH9 3HJ, UK \\ e-mail: copi@roe.ac.uk \\ ${ }^{3}$ Univ. Lyon, Univ. Lyon 1, ENSL, CNRS, LGL-TPE, 69622 Villeurbanne, France \\ ${ }^{4}$ Operations Department, European Space Astronomy Centre/ESA, PO Box 78, 28691 Villanueva de la Canada, Madrid, Spain
}

Received 24 July 2020 / Accepted 1 October 2020

\begin{abstract}
Aims. Observations of comet 67P/Churyumov-Gerasimenko were performed with MUSE at large heliocentric distances postperihelion between 3 and 7 March 2016. These observations are part of a simultaneous ground-based campaign aimed at providing broad-scale information about comet 67P to complement the ESA/Rosetta mission.

Methods. We obtained a total of 38 datacubes over five nights. We took advantage of the integral field unit nature of the instrument to carry out a simultaneous study of the spectrum of 67P's dust and its spatial distribution in the coma. We also looked for evidence of gas emission in the coma.

Results. We produced a high-quality spectrum of the dust coma over the optical range that could be used as a reference for future comet observations with this instrument. The slope of the dust reflectivity is of $10 \% / 100 \mathrm{~nm}$ over the $480-900 \mathrm{~nm}$ interval, with a shallower slope towards redder wavelengths. We used the Af $\rho$ to quantify the dust production and measure values of $65 \pm 4 \mathrm{~cm}$, $75 \pm 4 \mathrm{~cm}$, and $82 \pm 4 \mathrm{~cm}$ in the $V, R$, and $I$ bands, respectively. We detected several jets in the coma as well as the dust trail. Finally, using a novel method combining spectral and spatial information, we detected the forbidden oxygen emission line at $630 \mathrm{~nm}$. Using this line, we derived a water production rate of $1.5 \pm 0.6 \times 10^{26}$ molec. $\mathrm{s}^{-1}$, assuming all oxygen atoms come from the photo-dissociation of water.
\end{abstract}

Key words. techniques: imaging spectroscopy - comets: individual: 67P/Churyumo-Gerasimenko

\section{Introduction}

The ESA/Rosetta mission was the first mission to approach and follow a comet for a large part of its orbit, and to land on its nucleus. The Rosetta orbiter and the Philae lander were equipped with a series of instruments meant for in situ studies of the gas and dust coma surrounding comet 67P/ChuryumovGerasimenko (hereafter, 67P) and the properties of its nucleus. The mission provided unprecedented insights into the structure, composition, and activity evolution of 67P, as well as comets in general (Taylor et al. 2017).

To complement the mission, an ambitious ground-based campaign was set up, with dozens of telescopes across the globe and in space performing observations of the comet at the same time as the Rosetta mission (Snodgrass et al. 2017a). These observations probed the large-scale coma of 67P and provided context for the measurements performed by the Rosetta mission. More importantly, they provide a link to ground-based observations of a large number of comets that have been performed over the last century. As part of this campaign, observations were performed with the Multi Unit Spectroscopic Explorer (MUSE) instrument at the Very Large Telescopes (VLT). The observations were performed in March 2016, after the comet's perihelion passage, when the comet was moving away from the Sun. In this paper, we present the results of the MUSE observations of comet 67P.

\section{Observations and data reduction}

MUSE is an integral field unit spectrograph mounted on the UT4 telescope of the VLT in Chile (Bacon et al. 2010), nominally covering the $480-930 \mathrm{~nm}$ range. In the wide field mode, which was used for our observations, MUSE has a field of view (FoV) of $1^{\prime} \times 1^{\prime}$ covered without gaps. The instrument has a platescale of $0.2^{\prime \prime}$ pix $^{-1}$ and a spectral resolving power between 1770 (at $480 \mathrm{~nm}$ ) and 3590 (at $930 \mathrm{~nm}$ ). Observations of comet 67P were performed between 2016 March 3 and March 7. In total, 38 datacubes were obtained over five nights. For four of those cubes, the comet was not visible, or very badly centred, so we discarded their data. The sky during those five nights was either clear or photometric, but the seeing was variable, ranging between 0.6 and 2.3". For all observations, we used an exposure time of $600 \mathrm{~s}$, and the position angle of the instrument was set to $0^{\circ}$ (we did not apply any rotation between the exposures). At the time of the observations, even though the comet was still active, and thus extended, it did not fill the entire MUSE FoV. Because of this, no dedicated sky observations were performed. The observing circumstances are presented in Table 1.

The data reduction was performed using the ESO pipeline (Weilbacher et al. 2016), with the sky estimated from regions near the edge of the cubes that are free from comet contamination. In addition to the cube reconstruction, bias subtraction, flatfield correction, wavelength calibration, and sky subtraction, 
Table 1. Observing circumstances of the 67P MUSE campaign.

\begin{tabular}{lcccccc}
\hline \hline Date (UT) & $N$ & Exposure time $(\mathrm{s})$ & $r(\mathrm{au})$ & $\Delta(\mathrm{au})$ & Airmass & Phase angle $\left(^{\circ}\right)$ \\
\hline 2016-03-03 03h27-06h08 & 12 & 600 & 2.49 & 1.52 & $1.2-1.6$ & 6 \\
2016-03-04 05h34 & 1 & 600 & 2.50 & 1.53 & 1.2 & 6 \\
2016-03-05 04h50-06h29 & 8 & 600 & 2.51 & 1.53 & $1.2-1.3$ & 5 \\
2016-03-06 03h25-04h00 & 4 & 600 & 2.52 & 1.54 & $1.4-1.5$ & 5 \\
2016-03-07 04h39-07h38 & 13 & 600 & 2.52 & 1.54 & $1.3-1.6$ & 4 \\
\hline
\end{tabular}

the ESO MUSE pipeline also corrects for the telluric absorption and flux-calibrates the science data, using a standard star observed the same night as the science observations.

Even though the sky was estimated directly on the science cubes and subtracted by the pipeline, while examining the reduced cubes we noticed relatively strong sky residuals. In order to reduce those, we then used the ZAP (Zurich Atmosphere Purge) software (Soto et al. 2016). ZAP is a principal component analysis-based software that is designed to perform sky subtraction on integral field unit (IFU) data. The use of ZAP allows us to remove most of the sky residuals left after the pipeline reduction. As we discuss later in this paper, we also performed a full data reduction without applying any sky subtraction in order to search for forbidden oxygen emission lines in the coma of 67P.

As mentioned previously, the ESO pipeline performs a correction of the telluric absorption using a standard star observed on the same night as the science data. However, the standard stars are primarily used for flux calibration and are not optimal for telluric correction. Also, they are not always observed just after the science observations nor at the same airmass. Because of this, in the reduced cubes, there are residuals of the strong $\mathrm{O}_{2}$ telluric band around $760 \mathrm{~nm}$ when the telluric correction is performed with the pipeline. For all of the analysis focused on the 2D structure of the dust coma, and the detection of forbidden oxygen lines, this does not impact the quality of our measurements since those residuals are relatively constant over the field and we extracted the cubes over wavelength ranges that mostly avoid that region of the spectrum. However, in Sect. 3.1, we focus on the spectrum of the dust in the coma of $67 \mathrm{P}$ and, thus, the residual from the telluric correction could impact the quality of the spectrum presented. For the spectra presented in that section, we used the Molecfit software (Smette et al. 2015; Kausch et al. 2015) to perform a better telluric correction. Molecfit has proven to be very useful to provide an accurate telluric correction even when dedicated observations of a standard star are not available. We applied Molecfit directly on the extracted 1D spectra. The fit of the telluric features was performed for each extracted spectrum individually.

\section{Analysis}

\subsection{A reference dust spectrum}

We first studied the spectrum of comet $67 \mathrm{P}$ over the optical range. To do so, we extracted all the data cubes over a five-pixel radius $\left(1^{\prime \prime}\right)$ aperture around the comet optocenter. We chose such a small aperture so as to focus on the part of the coma where the signal is the strongest and to avoid having strong sky residuals in the presented spectrum. After the extraction, the spectra were corrected for the telluric features using the Molecfit software, as explained above. All 34 spectra were then median-combined to produce a high quality spectrum of the dust in the coma of
67P. This spectrum is shown in the upper part of Fig. 1. We do not see any emission band in the spectrum because of the large heliocentric distance and low activity level of the comet at the time of the observations. In the same part of this figure, we show a reference solar spectrum obtained using the SOLar SPECtrometer (SOLSPEC) instrument of the SOLAR payload onboard the International Space Station (ISS, Meftah et al. 2018), which was re-sampled to match the sampling of the 67P spectrum. Given the aperture we chose is very small, the spectrum shown in Fig. 1 probably contains a non-negligible contribution from the nucleus. For the purposes of comparison, we also extracted the dust spectrum over a $10^{\prime \prime}$ aperture, in which the dust coma dominates (blue dashed curve in the top part of Fig. 1). The two spectra match well, except for a small difference around $880 \mathrm{~nm}$. This region is severely affected by telluric absorption and has strong sky emissions, which might explain this difference. In general, we can say that the spectrum extracted over the $1^{\prime \prime}$ aperture is representative of the coma dust and we limit our discussion to this spectrum in the following text.

In the bottom part of Fig. 1, we divide the comet spectrum by the solar reference spectrum to compute the relative reflectance of the dust in the coma of 67P. The reflectance spectrum is normalised at $600 \mathrm{~nm}$. Overlaid to the reflectance spectrum measured with MUSE, we have the reflectance measured using the X-shooter spectrograph in November 2014 over the same wavelength range (Snodgrass et al. 2016). The reflectance spectra measured in November 2014 and March 2016 are consistent with each other, indicating that the dust reflectance as measured from the ground is similar at large heliocentric distance pre- and post-perihelion. We do not see any sign of absorption bands in the optical spectrum of 67P. We measure a spectral slope for the reflectance, or dust reddening, of $10 \% / 100 \mathrm{~nm}$ in the 480 $900 \mathrm{~nm}$ interval. We also notice that the slope becomes shallower at longer wavelengths. We measure a slope of $13 \% / 100 \mathrm{~nm}$ in the 500-700 nm interval but only of 5\%/100 nm in the 700$900 \mathrm{~nm}$ interval. This is fully consistent with what was reported in X-shooter observations performed in 2014, where values between $10 \% / 100 \mathrm{~nm}$ and 20\%/100 nm were given in the 550 to $1000 \mathrm{~nm}$ interval, with the shallower values corresponding to the red end of the wavelength range.

Our measurements are also consistent with in situ measurements of the dust from the ESA/Rosetta mission. Bertini et al. (2017) report a reddening in the interval 376-744 nm ranging between 11 and 14\%/100 nm from measurements with the OSIRIS cameras. Similarly, La Forgia et al. (2019) report slopes measured in the inner coma ranging from 12 to 16\%/100 nm between 480 and $649 \mathrm{~nm}$. Those slopes are similar to those measured for the nucleus of 67P, both from in situ and ground-based observations. Fornasier et al. (2015) report average slopes of $11-16 \% / 100 \mathrm{~nm}$ over $250-1000 \mathrm{~nm}$ with shallower slopes toward longer wavelengths, from observation of 

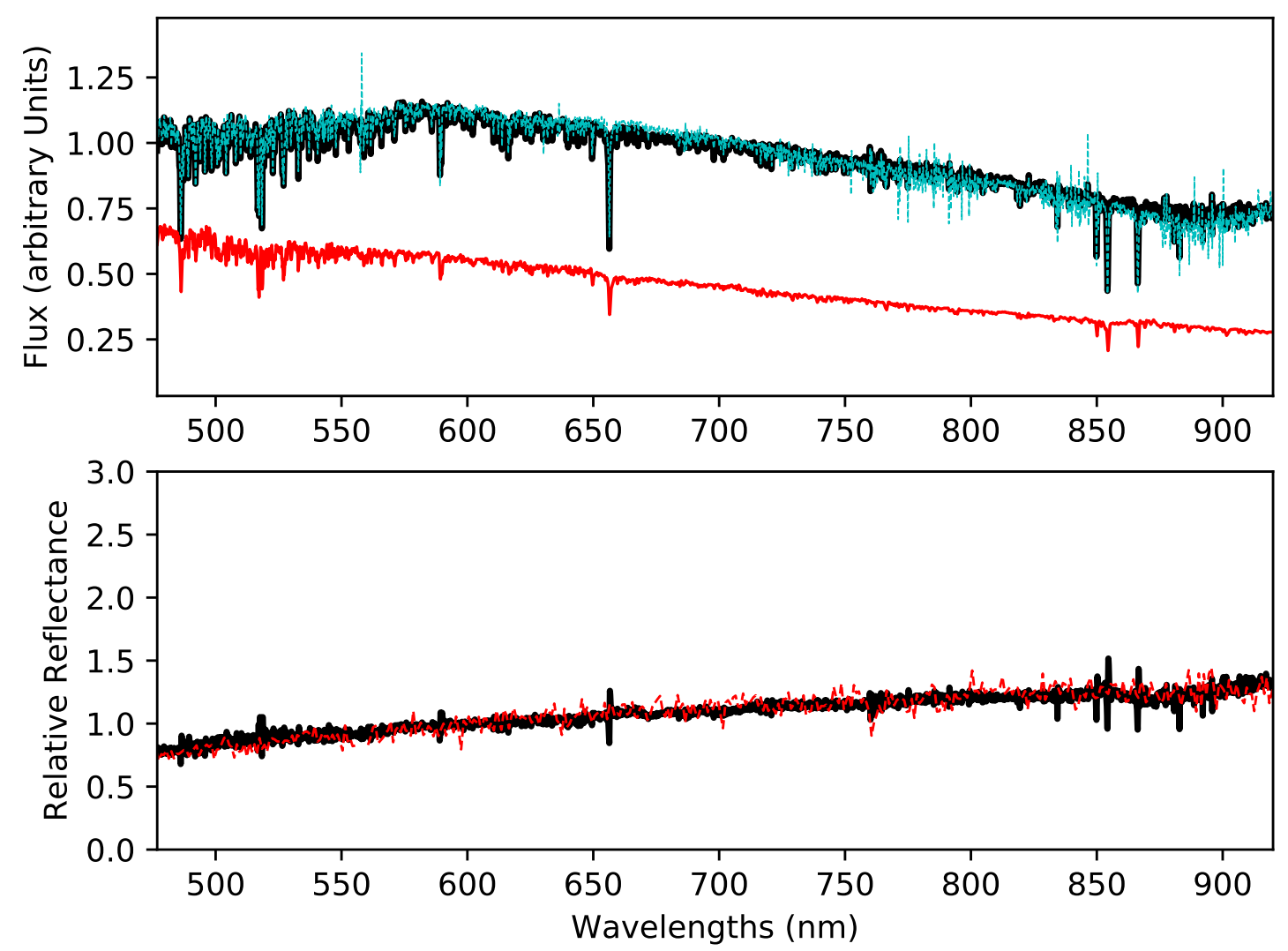

Fig. 1. Top: spectrum of 67P dust coma (black) together with the solar spectrum used to compute the reflectance spectrum (red). The blue dotted line is the spectrum extracted over a $10^{\prime \prime}$ aperture. The solar spectrum has been shifted arbitrarily over the $y$-axis for better visibility. Bottom: relative reflectance spectrum of 67P (black), compared to the one measured with X-shooter in November 2014 (red; Snodgrass et al. 2016).

67P's nucleus with the Rosetta/OSIRIS camera. From groundbased observations, Tubiana et al. (2011) measure a slope for the nucleus of $12 \pm 1 \% / 100 \mathrm{~nm}$ over $430-850 \mathrm{~nm}$ and slightly shallower in the 500-850 $\mathrm{nm}$ range. Finally, the dust reddening measured in the coma of $67 \mathrm{P}$ is consistent with what is measured usually in the coma of active comets, typically between 0 and $20 \% / 100 \mathrm{~nm}$, and with a shallower slope towards the near-IR (Solontoi et al. 2012; Jewitt \& Meech 1986).

\subsection{Dust coma morphology and activity}

For each cube in our dataset, we extracted maps over the bandpasses of the $V, R$, and $I$ Johnson-Cousins filters. All maps in the same bandpass were re-centred and then co-added. The resulting co-added maps are displayed in the top part of Fig. 2. The dust coma morphology is the same for all three bandpasses. It is asymmetrical, likely due to the presence of dust jets. To investigate the presence of jets further, we divided the dust maps by an azimuthal median profile (Samarasinha et al. 2013). Enhanced maps are displayed on the bottom part of Fig. 2. Here, we can clearly see two jets, which are most likely the cause of the apparent asymmetry of the dust coma. The first jet is located close to the anti-sunward direction, towards the south-west. The second jet is located about $90^{\circ}$ away, towards the south-east. Finally, we see a faint feature towards the sunward direction. Those jets are consistent with what was observed on previous passages and what is reported by Knight et al. (2017) and Snodgrass et al. (2017a) from observations at the same epoch. They are also consistent with the modelling of the pole orientation and active region location done by Vincent et al. (2013) prior to the Rosetta mission. In addition to the two jets mentioned above, we see an enhancement towards the North-West. This corresponds to the dust trail that was reported to be at least two degrees long at that epoch (Snodgrass et al. 2017a; Boehnhardt et al. 2016; Knight et al. 2017). We do not see changes of the coma morphology over the five nights of our observations, nor over a single night.

To constrain the comet activity at that time, we compute the Af $\rho$ value, which is a proxy for dust production, as defined by A'Hearn et al. (1984). We compute the $\operatorname{Af} \rho$ for the $V, R$, and $I$ bands over a $2500 \mathrm{~km}$ physical aperture. We use a $2500 \mathrm{~km}$ aperture instead of the more commonly used $10000 \mathrm{~km}$ aperture because the comet signal drops significantly at $10000 \mathrm{~km}$. We obtain values of $65 \pm 4 \mathrm{~cm}, 75 \pm 4 \mathrm{~cm}$, and $82 \pm 4 \mathrm{~cm}$ in the $V, R$, and $I$ bands, respectively. Those values have not been corrected for the phase angle effect and are consistent with those reported by Boehnhardt et al. (2016) at the same epoch, and they are comparable (even though slightly lower) to those reported by Knight et al. (2017) once the phase angle effect is taken into account. Using the Af $\rho$ values in the different bands, we can also compute the dust reflectivity gradient defined as (Jewitt \& Meech 1986):

$S^{\prime}=\frac{\operatorname{Af} \rho_{2}-\operatorname{Af} \rho_{1}}{\operatorname{Af} \rho_{2}+\operatorname{Af} \rho_{1}} \frac{20000}{\lambda_{2}-\lambda_{1}}$

where $\operatorname{Af} \rho_{\mathrm{x}}$ and the corresponding wavelength $\lambda_{x}$ are expressed in $\mathrm{cm}$ and $\mathrm{nm}$, respectively. For the $I-V$ combination, this gives a reflectivity gradient of $7 \pm 1 \% / 100 \mathrm{~nm}$, while it is of $11 \pm 2 \% / 100 \mathrm{~nm}$ for the $R-V$ and $7 \pm 1 \% / 100 \mathrm{~nm}$ for the $I-R$ combination. This confirms the trend outlined in Sect. 3.1 of higher reflectivity gradient at lower wavelengths. To check for 

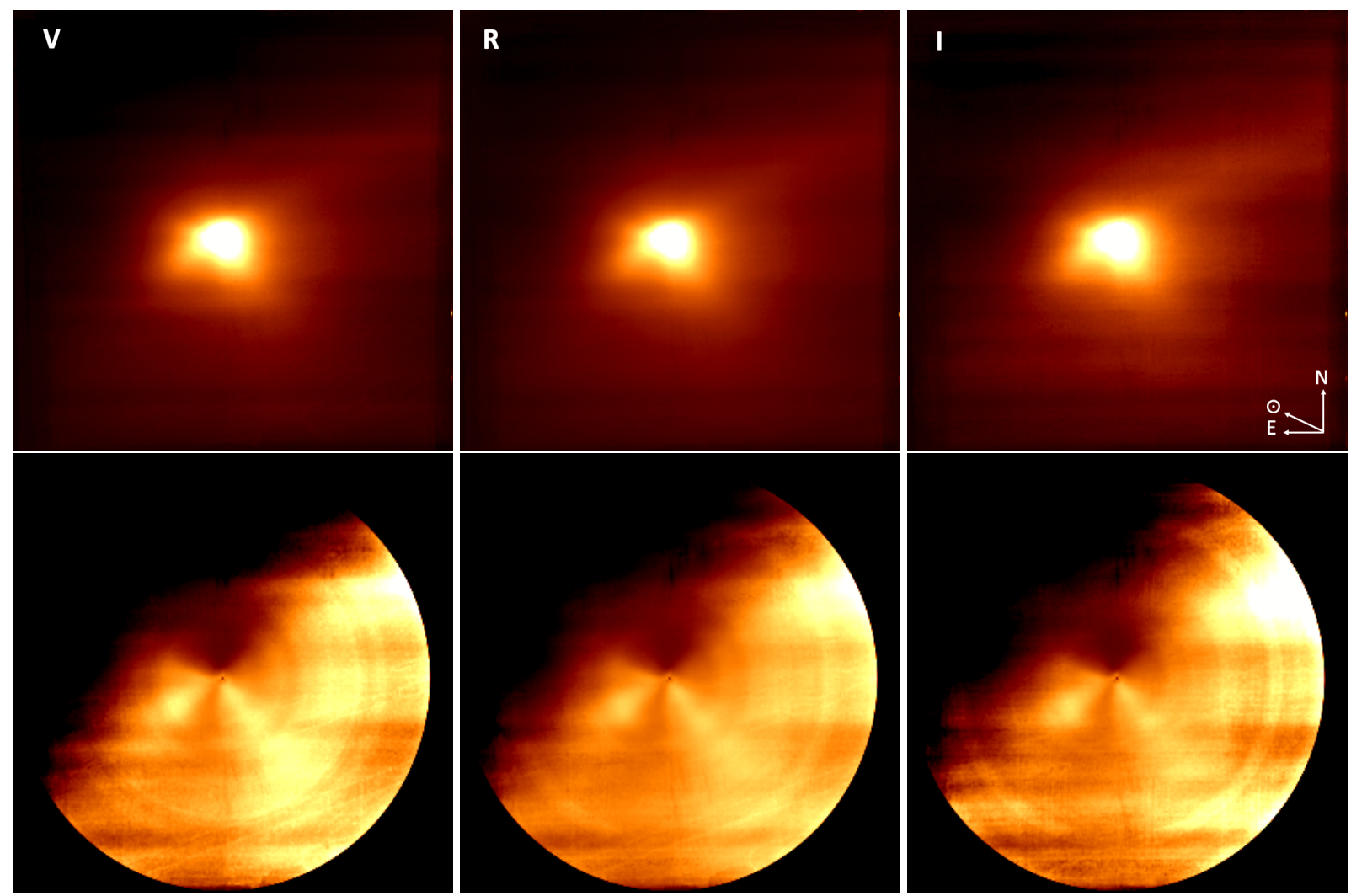

Fig. 2. Top: Combined maps of $67 \mathrm{P}$ in $V, R$, and $I$ bands. The maps are centred on the comet and the FoV is $1^{\prime} \times 1^{\prime}$. Bottom: $V$, $R$, and $I$ maps enhanced by dividing by an azimuthal median profile (Samarasinha et al. 2013).

trends with the aperture size, we computed the $\operatorname{Af} \rho$ in apertures of 5000, 7500, and $10000 \mathrm{~km}$. Within the error bars, we do not see significant changes in the Af $\rho$ or reflectivity gradient values with the aperture size in our data, as expected for a steady-state coma.

\subsection{Gas detection}

Among the features usually observed in the optical spectrum of comets, the oxygen forbidden line at $630 \mathrm{~nm}$ is one of the brightest. There are three forbidden oxygen lines that can be detected in the coma of comets at optical wavelengths: the green line at $557.7 \mathrm{~nm}$ and the red doublet at 630 and $636.4 \mathrm{~nm}$. Those lines are emitted by the decay of oxygen atoms in a metastable $\left({ }^{1} \mathrm{D}\right)$ or $\left({ }^{1} \mathrm{~S}\right)$ state. Exited atomic oxygen is mainly produced through the photo-dissociation of $\mathrm{H}_{2} \mathrm{O}, \mathrm{CO}, \mathrm{CO}_{2}$, or even $\mathrm{O}_{2}$ (see e.g. Cessateur et al. 2016). Observing forbidden oxygen lines then represents an opportunity to constrain the production of those potential parent species from optical observations. However, the cometary forbidden oxygen lines are often blended with the equivalent atmospheric lines, unless observed at high spectral resolution.

The spectral resolution of MUSE does not allow us to resolve the telluric and cometary lines for the geocentric velocity of comet $67 \mathrm{P}$ at the time of our observations. In theory, the sky subtraction should subtract the atmospheric contribution, allowing us to recover the cometary signal. However, the noise introduced by the subtraction of the very strong atmospheric features prevents us from detecting any cometary forbidden oxygen emission lines. At the time of our observations, the comet was at 2.5 au post-perihelion and was only weakly active, as confirmed by the fact that we do not detect any emission lines in the spectrum presented in Fig. 1. If the oxygen lines are present, they are thus very faint and masked by the noise introduced by the sky subtraction.

Therefore, we attempted to detect the forbidden oxygen lines using another method. We reduced the full dataset without performing any sky subtraction. For each spaxel of each cube, we then subtracted the continuum sky contribution and the dust contribution underlying the oxygen forbidden lines by defining continuum region on both sides of the red doublet and the green oxygen lines, fitting a line through those regions and subtracting it. Finally, we extracted the datacubes over a very narrow wavelength range centred on the wavelength of the three oxygen lines. In low and medium resolution spectra, forbidden oxygen lines can be contaminated by $\mathrm{NH}_{2}$ or $\mathrm{C}_{2}$ lines, but this is highly unlikely in the case of these observations since the comet is weakly active and no emission lines are detected in Fig. 1. The result of this process is maps of the flux contained in the forbidden lines at 557.7, 630, and 636.4 nm over the whole MUSE FoV. Since the atmospheric and cometary lines cannot be resolved by MUSE, the maps contain the sum of the atmospheric and potential cometary contribution. All the maps are re-centred so that the optocenter of the comet is placed at the same position. We average all 34 maps for each line, performing a 3- $\sigma$ clipping. The result in shown in Fig. 3.

In those maps, we expect the atmospheric contribution to be relatively uniform over the $1^{\prime} \times 1^{\prime}$ FoV of MUSE. The cometary contribution, on the other side, would be concentrated around the optocenter of the comet, with a limited spatial extension since the states responsible for the emission lines are metastable states. In Fig. 3, we can see that for the 557.7 and $636.4 \mathrm{~nm}$ lines, the maps are mostly uniform over the whole FoV. Small 

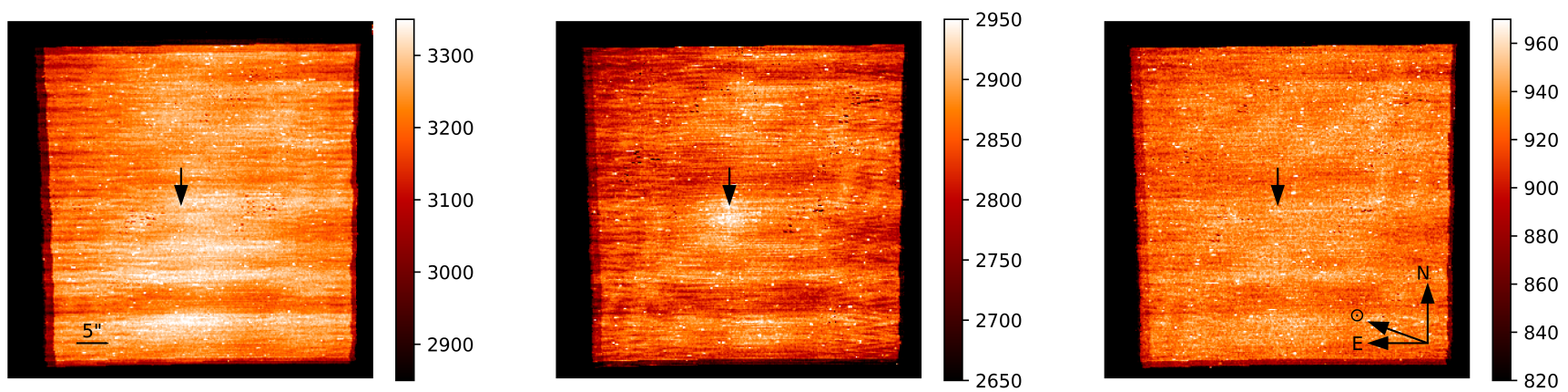

Fig. 3. Combined maps of the sky+comet flux at the wavelengths of the three forbidden oxygen lines at $557.7 \mathrm{~nm}($ left $), 630 \mathrm{~nm}($ middle), and $636.4 \mathrm{~nm}$ (right). North is up, east is to the left, and the black arrow in the centre points to the position of the comet optocenter. The unit of the color bars is $10^{-20} \mathrm{erg} \mathrm{s}^{-1} \mathrm{~cm}^{-2}$.

variations are observed but they most likely come from nonoptimal corrections of the detector-to-detector effects. We thus conclude that the signal in those maps comes from the atmospheric oxygen lines. In the $630 \mathrm{~nm}$ map, however, in addition to inhomogeneities similar to those seen in the other two maps, we have a clear over-density located around the position of the comet optocenter. This indicates that in addition to the atmospheric line, we detect a signal from the cometary forbidden oxygen line at $630 \mathrm{~nm}$. It is not surprising that we only detect the $630 \mathrm{~nm}$ line since it is the strongest of the three oxygen lines. The signal we measure in the comet aperture for the $630 \mathrm{~nm}$ map is barely 3- $\sigma$ above the sky background variation (measured from four adjacent sky apertures, see more later in this paper). Since the ratio between the $630 \mathrm{~nm}$ and $636.4 \mathrm{~nm}$ maps is expected to be three (as it is the case for the background in the two maps, see Fig. 3), this is then consistent with the fact that we do not detect significant cometary signal in the $636.4 \mathrm{~nm}$ map. We note that the [OI] cometary signal is enhanced towards the south, similarly to what is observed for the dust. However, we do not believe the signal is due to residuals from the dust continuum. Indeed, the technique used for the dust subtraction is the same for all three lines and it is only the $630 \mathrm{~nm}$ map (with the strongest oxygen feature) that shows an over-density around the optocenter. Control maps built using a wavelength range adjacent to the $630 \mathrm{~nm}$ line do not show a similar signal.

The cometary signal we detect in the $630 \mathrm{~nm}$ map is faint, but we use this signal to estimate the water production rate of the comet at that time. The $630 \mathrm{~nm}$ line comes from the decay of atomic oxygen in $\left({ }^{1} \mathrm{D}\right)$ state. As mentioned before, oxygen in that state is mainly produced by the photo-dissociation of $\mathrm{H}_{2} \mathrm{O}, \mathrm{CO}$, $\mathrm{CO}_{2}$, or $\mathrm{O}_{2}$. For comets at 1 au from the Sun, $\mathrm{H}_{2} \mathrm{O}$ photodissociation is the dominant source for producing metastable oxygen, but at distances above $2.5 \mathrm{au}$, other molecules such as $\mathrm{CO}$ and $\mathrm{CO}_{2}$ start to contribute (Decock et al. 2013; McKay et al. 2015). Longterm measurements of the production rate of all four species of interest derived from the ROSINA instrument onboard Rosetta are presented by Laeuter et al. (2020) and Combi et al. (2020). At the time of our observations, the $\mathrm{CO}$ and $\mathrm{O}_{2}$ production rates are more than a factor of ten and 100 lower than that of $\mathrm{H}_{2} \mathrm{O}$, respectively. We can thus assume that those species will contribute little to the production of metastable oxygen. The $\mathrm{CO}_{2}$ production rate is only about a factor of two to four lower than the $\mathrm{H}_{2} \mathrm{O}$ production rate. The emission rate for $\mathrm{O}\left({ }^{1} \mathrm{D}\right)$ production from $\mathrm{CO}_{2}$ is 1.5 times higher than that of $\mathrm{H}_{2} \mathrm{O}$ (Bhardwaj \& Raghuram 2012). Taking this into account, a significant part (up to half) of $\mathrm{O}\left({ }^{1} \mathrm{D}\right)$ atoms could be produced by the photo-dissociation of $\mathrm{CO}_{2}$. For the measurements presented below, we assume that water is the main source for the production of metastable oxygen, thus, the water production rate we derive might be overestimated.

In order to derive water production rates, we follow the procedure described in Schultz et al. (1992) and Morgenthaler et al. (2001). We consider a photo-chemical model that includes the following three reactions,

$$
\begin{aligned}
& \mathrm{H}_{2} \mathrm{O}+\mathrm{h} v \longrightarrow \mathrm{H}_{2}+\mathrm{O}\left({ }^{1} \mathrm{D}\right), \\
& \mathrm{H}_{2} \mathrm{O}+\mathrm{h} v \longrightarrow \mathrm{H}+\mathrm{OH}, \\
& \mathrm{OH}+\mathrm{h} v \longrightarrow \mathrm{H}+\mathrm{O}\left({ }^{1} \mathrm{D}\right),
\end{aligned}
$$

and the water production rate is given by:

$$
Q\left(\mathrm{H}_{2} \mathrm{O}\right)=\frac{Q([\mathrm{OI}])}{B R 1+(B R 2)(B R 3)},
$$

where BR1 and BR2 are the branching ratios for reactions (1) and (2) (Huebner et al. 1992) (equal to 0.05 and 0.855 for the quiet Sun, respectively) and BR3 is the branching for reaction (3) from Morgenthaler et al. (2001) (equal to 0.094). The production rate of oxygen atoms in $\left({ }^{1} \mathrm{D}\right)$ state, $\mathrm{Q}([\mathrm{OI}])$, can be obtained from:

$Q([\mathrm{OI}])=\frac{4}{3} 4 \pi \Delta^{2} I_{630} \mathrm{AC}$,

where $\Delta$ is the comet geocentric distance (in $\mathrm{cm}$ ), $I_{630}$ is the intensity of the [OI] emission (in photons $\mathrm{s}^{-1} \mathrm{~cm}^{-2}$ ) and $\mathrm{AC}$ is the aperture correction factor correcting for the $[\mathrm{OI}]$ emission not encompassed in the aperture.

We measure the comet flux in the $630 \mathrm{~nm}$ map using a 25 pixels $\left(5^{\prime \prime}\right)$ radius circular aperture centred on the comet optocentre. In order to measure and subtract the sky flux, we measure the flux in four $5^{\prime \prime}$ apertures located at four different positions with respect to the comet optocenter $((+50$ pix,+50 pix $),(+50$ pix, -50 pix $),(-50$ pix,+50 pix $),(-50$ pix,-50 pix $))$, then we compute the average and subtract it from the flux measured in the comet aperture. Given the faintness of the cometary emission, the main uncertainty in the determination of the water production rate comes from the measurement of the sky. To estimate this uncertainty, we used the upper and lower values measured in the individual sky apertures. The resulting intensity of the comet $630 \mathrm{~nm}$ line is $1.8 \pm 1.0 \times 10^{-3}$ photons $\mathrm{cm}^{-2} \mathrm{~s}^{-1}$. The aperture we used encompasses all the visible emission from the comet, so that we set the aperture correction factor to 1 . This results in a water production rate of $1.5 \pm 0.7 \times 10^{26}$ molec s $^{-1}$. This represents the actual water production rate only if all the oxygen atoms in $\left({ }^{1} \mathrm{D}\right)$ state are produced by the photo-dissociation of water. 

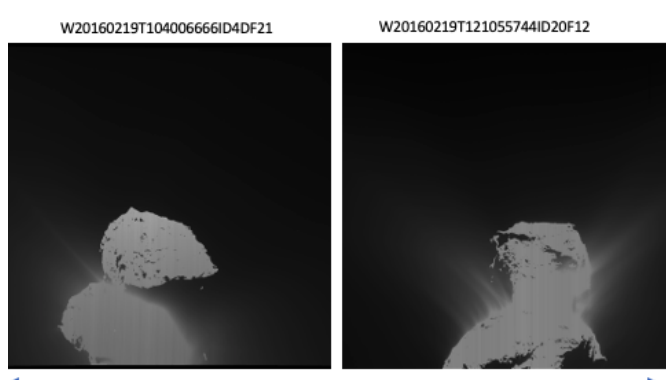

ESA/Rosetta Osiris WAC images from $19 / 02 / 2016$ outburst
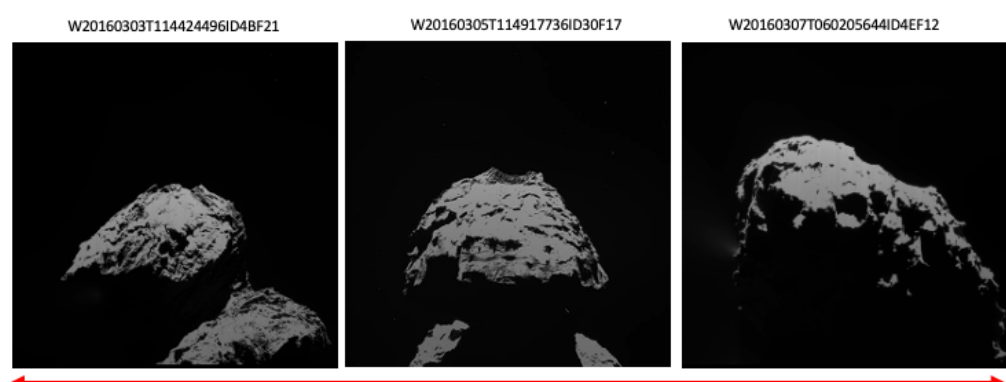

ESA/Rosetta Osiris WAC images from the same epoch as MUSE observations (between 03/03/2016 and 07/03/2016)

Fig. 4. Images of the nucleus of 67P taken by the OSIRIS instrument on-board Rosetta during the 19/02/2016 outburst and at the same epoch as the MUSE observations presented here. Credit: ESA/Rosetta/MPS for OSIRIS Team MPS/UPD/LAM/IAA/SSO/INTA/UPM/DASP/IDA.

No other measurement of the comet water production rate was reported from ground-based observations at a similar epoch because the comet was too faint and weakly active. In situ measurements made with the ROSINA DFMS instrument determined a water production rate around $2.3 \times 10^{26}$ molec s$^{-1}$ at the same heliocentric distance (Hansen et al. 2016). Similar values are reported by Combi et al. (2020) and Laeuter et al. (2020) using the same instrument. Biver et al. (2019) report a water production rate of $8.5 \pm 2.5 \times 10^{25}$ molec s$^{-1}$ from measurements with the MIRO instrument. Given that $\mathrm{CO}_{2}$ could potentially contribute up to half of the $630 \mathrm{~nm}$ [OI] line brightness, the actual water production rate we measure could be as low as $0.7 \times 10^{26}$ molec s ${ }^{-1}$, which is in very good agreement with the Biver et al. (2019) measurement. Given the difference in the methods, scale of the observations, and models used, our measurement is close to those reported using Rosetta instruments, in particular, the MIRO instrument.

\section{Discussion and conclusions}

Comet 67P was observed with the MUSE IFU over five nights between 3 and 7 March 2016 when the comet was at 2.5 au from the Sun and 1.5 au from the Earth. The spectrum of the dust coma of $67 \mathrm{P}$ presented here is of high quality. It has a good signal-to-noise ratio given the faintness and the distance of the comet at the time of our observations. Thanks to the use of the Molecfit software, it is little affected by telluric features at nearIR wavelengths, which is not the case for most comet spectra in the 800-900 $\mathrm{nm}$ range. It matches very well with other groundbased observations of 67P, as well as with in situ measurements from the Rosetta spacecraft. It is also representative of what is usually observed for active comets in general. Finally, it does not contain gas emission features above the noise level (there is some [OI] signal present and merged with the sky line; see more below). For all those reasons, this spectrum could be used in the future as a "template" dust spectrum to help perform the subtraction of the dust-reflected continuum for observations of other comets with the MUSE IFU. This spectrum could replace the observation of solar analogues since, so far, very few good solar analogues have been observed with MUSE. The dust reddening can vary from one comet to another, so this spectrum would need to be corrected from any slope difference between the target and 67P. Nonetheless, this dust-only spectrum of $67 \mathrm{P}$ represents a good tool to help in the analysis of future comet observations with MUSE. The morphology of the coma, the dust activity, and the dust reflectivity gradient as measured with MUSE are consistent with other measurements performed with ground-based telescopes at the same epoch as well as with in situ measurements from the Rosetta mission.

About two weeks before our observations, on 2 February 2016, an outburst was detected by several instruments on board the Rosetta orbiter (Grün et al. 2016). A similar but less intense event was also detected in NAVCAM images on 1 March 2016. Since the outburst(s) happened shortly before our observations, we could have expected to detect some indication of such an event in our observations, in terms of either the activity level or coma morphology. However, we did not detect any sign of the outburst in the MUSE data presented here. In Fig. 4, we show images obtained with the OSIRIS WAC on-board Rosetta. In the left part, gas jets are easily detected during the 19 February outburst. The right side shows the comet at the same epoch as our observations. No gas jets are visible. This is consistent with the comet having reverted back to a quieter state and the fact that we do not detect the outburst in the MUSE observations.

Through a careful reprocessing and separation of comet and sky signals spatially (rather than by resolving them spectroscopically), we detected the $630 \mathrm{~nm}$ forbidden oxygen line and derive a water production rate of $1.5 \pm 0.7 \times 10^{26}$ molec s$^{-1}$ in the coma of $67 \mathrm{P}$, if all [OI] atoms in $\left({ }^{1} \mathrm{D}\right)$ state are produced by water. This value is consistent with the Rosetta measurements (Hansen et al. 2016; Combi et al. 2020; Laeuter et al. 2020; Biver et al. 2019). It is the only measurement of the water production rate of $67 \mathrm{P}$ at such a large heliocentric distance from remote observations. Ground-based detection of water (or water products) in the coma of 67P only happened close to perihelion (Snodgrass et al. 2017a), so this point is extremely valuable with regard to comparing measurements and models from the Rosetta spacecraft to ground-based measurements over a larger portion of the comet orbit. In general, detecting water at such low production rate for comets at 2.5 au from the Sun is extremely difficult (see e.g. discussion on water detection in Snodgrass et al. 2017b). In the past, MUSE had been shown to hold a great potential in the study of species parentage in the coma of brighter comets (Opitom et al. 2019). Our present work demonstrates the efficiency of MUSE in detecting low levels of water production around distant solar system bodies. This opens up future opportunities at a time when an intensive search for evidence of water ice in the main asteroid belt and elsewhere in the solar system is underway.

Acknowledgements. Based on observations made with ESO Telescopes at the La Silla Paranal Observatory under programme 096.C-0160(A). Datasets of the ESA/Rosetta OSIRIS instrument have been downloaded from the ESA Planetary Science Archive. The authors acknowledge the OSIRIS Principal Investigator H. Sierks (MPS, Goettingen, Germany) and the OSIRIS Team for providing images and related datasets and the ESA Rosetta Project for enabling the science of the mission. 


\section{References}

A'Hearn, M. F., Schleicher, D. G., Millis, R. L., Feldman, P. D., \& Thompson, D. T. 1984, AJ, 89, 579

Bacon, R., Accardo, M., Adjali, L., et al. 2010, Proc. SPIE, 7735, 773508

Bertini, I., La Forgia, F., Tubiana, C., et al. 2017, MNRAS, 469, S404

Bhardwaj, A., \& Raghuram, S. 2012, ApJ, 748, 13

Biver, N., Bockelée-Morvan, D., Hofstadter, M., et al. 2019, A\&A, 630, A19

Boehnhardt, H., Riffeser, A., Kluge, M., et al. 2016, MNRAS, 462, S376

Cessateur, G., de Keyser, J., Maggiolo, R., et al. 2016, J. Geophys. Res. Space Phys., 121, 804

Combi, M., Shou, Y., Fougere, N., et al. 2020, Icarus, 335, 113421

Decock, A., Jehin, E., Hutsemékers, D., \& Manfroid, J. 2013, A\&A, 555, A34

Fornasier, S., Hasselmann, P. H., Barucci, M. A., et al. 2015, A\&A, 583, A30

Grün, E., Agarwal, J., Altobelli, N., et al. 2016, MNRAS, 462, S220

Hansen, K. C., Altwegg, K., Berthelier, J. J., et al. 2016, MNRAS, 462, S491

Huebner, W. F., Keady, J. J., \& Lyon, S. P. 1992, Ap\&SS, 195, 1

Jewitt, D., \& Meech, K. J. 1986, ApJ, 310, 937

Kausch, W., Noll, S., Smette, A., et al. 2015, A\&A, 576, A78

Knight, M. M., Snodgrass, C., Vincent, J.-B., et al. 2017, MNRAS, 469, S661

Laeuter, M., Kramer, T., Rubin, M., \& Altwegg, K. 2020, ArXiv e-prints [arXiv:2006.01750]
La Forgia, F., Lazzarin, M., Bodewits, D., et al. 2019, EPSC-DPS Joint Meeting 2019, EPSC-DPS2019-1442

McKay, A. J., Cochran, A. L., DiSanti, M. A., et al. 2015, Icarus, 250, 504

Meftah, M., Damé, L., Bolsée, D., et al. 2018, A\&A, 611, A1

Morgenthaler, J. P., Harris, W. M., Scherb, F., et al. 2001, ApJ, 563, 451

Opitom, C., Yang, B., Selman, F., \& Reyes, C. 2019, A\&A, 628, A128

Samarasinha, N. H., Martin, M. P., \& Larson, S. M. 2013, Cometary Coma Image

Enhancement Facility, http://www.psi.edu/research/cometimen

Schultz, D., Li, G. S. H., Scherb, F., \& Roesler, F. L. 1992, Icarus, 96, 190

Smette, A., Sana, H., Noll, S., et al. 2015, A\&A, 576, A77

Snodgrass, C., Jehin, E., Manfroid, J., et al. 2016, A\&A, 588, A80

Snodgrass, C., A’Hearn, M. F., Aceituno, F., et al. 2017a, Phil. Trans. R. Soc. London Ser. A, 375, 20160249

Snodgrass, C., Agarwal, J., Combi, M., et al. 2017b, A\&ARv, 25, 5

Solontoi, M., Ivezić, Ž., Jurić, M., et al. 2012, Icarus, 218, 571

Soto, K. T., Lilly, S. J., Bacon, R., Richard, J., \& Conseil, S. 2016, MNRAS, 458, 3210

Taylor, M. G. G. T., Altobelli, N., Buratti, B. J., \& Choukroun, M. 2017, Phil. Trans. R. Soc. London Ser. A, 375, 20160262

Tubiana, C., Böhnhardt, H., Agarwal, J., et al. 2011, A\&A, 527, A113

Vincent, J. B., Lara, L. M., Tozzi, G. P., Lin, Z. Y., \& Sierks, H. 2013, A\&A, 549, A121

Weilbacher, P. M., Streicher, O., \& Palsa, R. 2016, Astrophys. Source Code Libr. [record ascl:1610.004] 